\title{
Planetary Metabolism: Linking Solid \\ Interiors to Gaseous Biosignatures via Bioinorganic Chemistry \\ JENNIFER B GLASS
}

Georgia Institute of Technology

Presenting Author: jennifer.glass@eas.gatech.edu

The next few decades hold exhilarating potential for discovery of life beyond Earth and alarming threats for the future of Earth's habitability for Homo sapiens. The actions or inactions of industrial countries to limit carbon emissions will determine the trajectory of our planet's climate for several millennia. Extraordinary times mandate extraordinary measures, tasking us to emerge from our scientific niches and apply our disciplinary knowledge to the farthest reaches of the universe and the greatest problems facing our own planet. This session challenges us to develop a predictive theory of how planetary interiors and exteriors co-evolve. In this talk, I will highlight a few biochemical links that wed the Earth's solid interior and gaseous exterior and were likely key to the development and maintenance of habitability on Earth. Oxygen and methane are two gases in Earth's atmosphere whose co-existence in other planetary atmospheres may robustly indicate the activity of life as we know it. Indeed, the production of both oxygen and methane is almost completely biological today and through Earth history. Underpinning the perpetual production of oxygen and methane over billions of years are enzymes containing metal cofactors: a manganese cluster for photosynthetic splitting of water to yield oxygen gas, and a nickel-porphyrin cofactor for methane production in certain anaerobic archaea. These cofactors are identical in all organisms that perform this biochemistry, suggesting that life on Earth evolved one, and apparently only one, biochemical method for making large fluxes of each of these gases. (There are a few rare biotic and abiotic routes to making oxygen and methane, but these are insignificant on a global scale on Earth today and likely throughout its history.) This talk poses the question of whether we can predict the existence of oxygenic photosynthesis and/or methanogenesis on exoplanets based on the composition of their stellar spectra and, in particular, the relative abundance of first-row transition metals that are absolutely essential for metallocofactors in the enzymes on Earth that catalyze the reactions at the heart of biogeochemical cycles, and thereby link the solid and gaseous planetary spheres. 